\title{
Resource Allocation for Delay Differentiated Traffic in Multiuser OFDM Systems
}

\author{
Meixia Tao, Ying-Chang Liang and Fan Zhang
}

\begin{abstract}
Most existing work on adaptive allocation of subcarriers and power in multiuser orthogonal frequency division multiplexing (OFDM) systems has focused on homogeneous traffic consisting solely of either delay-constrained data (guaranteed service) or non-delay-constrained data (best-effort service). In this paper, we investigate the resource allocation problem in a heterogeneous multiuser OFDM system with both delay-constrained (DC) and non-delay-constrained (NDC) traffic. The objective is to maximize the sum-rate of all the users with NDC traffic while maintaining guaranteed rates for the users with DC traffic under a total transmit power constraint. Through our analysis we show that the optimal power allocation over subcarriers follows a multi-level water-filling principle; moreover, the valid candidates competing for each subcarrier include only one NDC user but all DC users. By converting this combinatorial problem with exponential complexity into a convex problem or showing that it can be solved in the dual domain, efficient iterative algorithms are proposed to find the optimal solutions. To further reduce the computational cost, a low-complexity suboptimal algorithm is also developed. Numerical studies are conducted to evaluate the performance the proposed algorithms in terms of service outage probability, achievable transmission rate pairs for DC and NDC traffic, and multiuser diversity.
\end{abstract}

\section{Index Terms}

Orthogonal frequency division multiplexing (OFDM), constant-rate transmission, variable-rate transmission, power control, convex optimization, water filling.

\section{INTRODUCTION}

Future broadband wireless networks are expected to support a wide variety of communication services with diverse quality-of-service (QoS) requirements. Applications such as voice transmission and real-time video streaming are very delay-sensitive and need guaranteed

This work was presented in part at the IEEE International Conference on Communications, Istanbul, Turkey, June 2006. The corresponding author is Meixia Tao, who is with the department of Electrical and Computer Engineering, National University of Singapore, Singapore 117576. Email: mxtao@nus.edu.sg. 
throughput. On the other hand, applications like file transfer and email services are relatively delay tolerant so variable-rate transmission is acceptable. From the physical layer point of view, transmission of delay-tolerant or non-delay-constrained (NDC) traffic can be viewed as an ergodic capacity problem [1], where maximizing the long-term average transmission rate is the goal. Thus, wireless resources, such as transmission power and frequency bandwidth, can be dynamically allocated so as to exploit the time or frequency selectivities of broadband wireless fading channels. Likewise, transmission of delay-sensitive or delay-constrained (DC) traffic can be regarded as a delay-limited capacity problem [2] in which a constant transmission rate should be maintained with probability one regardless of channel variations. In this case, it is desirable to allocate more transmission power and frequency bandwidth when the channel experiences deep fade and to allocate less resources when the channel is under favorable conditions. We investigate in this work resource allocation in a broadband wireless network that supports simultaneous transmission of users with delay differentiated traffic. Our focus is on the formulation of an analytical framework from the physical layer perspective as well as the design of efficient and practical algorithms.

Multicarrier transmission in the shape of orthogonal frequency division multiplexing (OFDM) is a leading technique to provide spectrally efficient modulation as well as user multiplexing in future wireless systems. With OFDM technique, the broadband wireless channel is divided into a set of orthogonal narrowband subcarriers. In a single user system, since the channel frequency responses are different at different subcarriers, the system performance can be significantly enhanced by adapting the transmission parameters such as modulation, coding, and power over each subcarrier. For instance, the transmitter can send at higher transmission rates over subcarriers with better channel condition while lower rates or no data over subcarriers in deep fade. This follows the well-known water-filling principle. In a multiuser system, different subcarriers can be allocated to different users to provide a multiple access method, also known as OFDMA. As the channels on each subcarrier are likely independent for different users, the subcarriers experiencing deep fade for one user may not be in deep fade for other users. As a result, each subcarrier could be in good condition for some users in a multiuser OFDM system. By adaptively allocating the subcarriers among multiple users based on instantaneous channel information, multiuser diversity can be utilized to boost the overall system efficiency.

Adaptive resource allocation in multiuser OFDM systems has focused on homogeneous traffic only. In such systems, the traffic consists solely of either DC data requiring constant- 
rate transmission [3], or variable-rate NDC data which can be served in a best-effort manner [4]-[6]. For systems with pure DC traffic, the problem is to minimize the total transmit power while satisfying a basic transmission rate for each user, which is often referred to as margin adaption [7]. In [3], an iterative algorithm was proposed to allocate each user a set of subcarriers and then determine the power and rate for each user on its allocated subcarriers. For systems with pure NDC traffic, the problem is often formulated as maximizing the sumrate of the system subject to a total transmit power constraint. This formulation is also known as rate adaptation [7]. In [4], it was shown that the total sum-rate of a multiuser OFDM system is maximized when each subcarrier is allocated to the user with the best channel gain for that subcarrier. The total transmit power is then distributed over the subcarriers using the water-filling algorithm. This result holds, however, only for single-antenna systems. It is no longer optimal when multiple antennas are deployed at the base station due to the spatial multiplexing gain [5], [6], [8]. Other problem formulations for systems with pure NDC traffic take user fairness into account. For example, [9] studied the max-min criterion which aims to maximize the transmission rate of the bottleneck user. In [10], it was proposed to maintain proportional rates among users for each channel realization. A utility-function based optimization framework to balance system efficiency and user fairness was also discussed in [11].

In this paper, we consider the subcarrier and power allocation problem in a heterogeneous multiuser OFDM system where DC and NDC traffic is supported simultaneously. Users in the system are classified into DC users and NDC users based on their traffic delay requirements. We assume that the total transmit power from the base station is fixed. Our objective is to maximize the sum-rate of all the NDC users while maintaining the basic transmission rates of all the DC users over every transmission frame. A similar problem was studied in [12]. However, it assumed static subcarrier allocation so only the transmit power adaptation was discussed. Our work, instead, considers joint subcarrier and power adaptation in multiuser OFDM systems, which is one step forward of the previous work. This multiuser subcarrier and power allocation problem is a mixed integer programming problem, the complexity of which increases exponentially with the number of subcarriers. To make the problem more tractable, we transform it into a convex programming problem by introducing time-sharing variables. We show that, for a given subcarrier assignment, the optimal power distribution is achieved by multi-level water-filling. In particular, the water level of each DC user depends explicitly on the channel gains of its assigned subcarriers and its basic rate requirement, 
and can differ from one another. On the other hand, the water levels of all NDC users are the same. We also show that, for the optimal subcarrier assignment, the set of valid user candidates competing for each subcarrier consists of all the users from the DC group and one from the NDC group with the best channel gain. Using these properties, we propose an efficient iterative algorithm to compute the optimal solution numerically. Alternatively, the original problem is solved in the dual domain by using dual decomposition. It is shown that the dual updates can be done efficiently using an ellipsoid algorithm. In addition, we present a suboptimal algorithm with linear complexity in the number of subcarriers and the number of users.

The rest of this paper is organized as follows. In Section II we introduce the system model and describe the problem formulation. In Section III we formulate the resource allocation problem as a convex optimization problem by using time-sharing technique and present analytical frameworks of the optimal solution. An iterative algorithm to search the optimal solution is also presented. In Section IV, we attempt to solve the problem using dual approach. A low-complexity suboptimal algorithm is given in Section V. In Section VI, we present numerical results of our proposed algorithms in a multiuser OFDM system. Finally conclusion and discussions are given in Section VII.

\section{System Model And Problem Formulation}

We consider the downlink of a multiuser OFDM system with block diagram shown in Fig. 1, The system consists of $K$ mobile users. The first $K_{1}$ users have DC traffic, which requires a constant transmission rate of $R_{k}\left(k=1, \ldots, K_{1}\right)$ bits per OFDM symbol, respectively. The traffic of the remaining $K-K_{1}$ users has no delay constraint and can be delivered in a best-effort manner. Note that $K$ is the number of users that are scheduled for transmission during a certain transmission interval. The total number of users in a practical system may be much larger than $K$ and, hence, other multiple access techniques such as time-division multiple access (TDMA) are needed in conjunction with OFDMA. The data streams from the $K$ users are serially fed into the encoder block at the base station transmitter. The total channel bandwidth is $B \mathrm{~Hz}$ and is divided into $N$ orthogonal subcarriers, which are shared among the $K$ users. The transmission is on a time-frame basis, where each frame consists of multiple OFDM symbols. The fading coefficients of all users are assumed to remain unchanged within each transmission frame but can vary from one frame to another. All channel information is assumed perfectly known at the central controller, which can 
be embedded with the base station. Typically, the channel information can be collected by estimating it at each user terminal and sending it to the base station via a feedback channel, or through channel estimation of the uplink in a time-division duplex system. Based on the instantaneous channel inputs, the central controller allocates different subcarriers to different users and determines the amount of power/bits to be transmitted on each subcarrier through the subcarrier and power/bit allocation algorithm. The resulting allocation information is used to configure the encoder block at the base station transmitter and to facilitate the subcarrier selector and decoder at each user receiver. Note that this allocation information may be sent to each user via a separate channel. The output data symbols from the encoder are then modulated by the inverse fast Fourier transform (IFFT). Guard interval is inserted to ensure orthogonality between OFDM symbols. The total transmit power from the base station is fixed and is given by $P_{T}$.

The broadband wireless channel between the base station and each user terminal is assumed to be frequency-selective Rayleigh fading. However, the channel in each subcarrier is narrow enough to experience flat fading. Let $r_{k, n}$ denote the transmission rate of user $k$ on subcarrier $n$ in bits per OFDM symbol. It depends on the channel gain $h_{k, n}$ and the allocated power $P_{k, n}$ of user $k$ on subcarrier $n$. In general, $r_{k, n}$ can be expressed as

$$
r_{k, n}=\log _{2}\left(1+\frac{P_{k, n}\left|h_{k, n}\right|^{2}}{\Gamma N_{0} B / N}\right)
$$

where $N_{0}$ is the power spectral density of additive white Gaussian noise and $\Gamma$ is a constant, usually called the signal-to-noise ratio (SNR) gap [7]. When instantaneous mutual information is used to characterize the achievable transmission rate, we have $\Gamma=1(0 \mathrm{~dB})$. If practical signal constellations are used, $\Gamma$ is a constant related to a given bit-error-rate (BER) requirement. For example, when uncoded QAM modulation is used we have $\Gamma=-\ln (5 \cdot \mathrm{BER}) / 1.5$. In general, the gap serves as a convenient mechanism for analyzing the difference between the SNR needed to achieve a certain data rate for a practical system and the theoretical limit. Throughout this paper we use (1) as a unified form to characterize both the theoretical mutual information and practical transmission rate.

The problem we consider here is to optimize the allocation of subcarriers and power under the total transmit power constraint so as to maximize the sum-rate of all the $K-K_{1}$ NDC users while satisfying the individual rate requirement for each of the $K_{1}$ DC users. 
Mathematically, the given problem can be formulated as

$$
\begin{aligned}
\max _{\left\{\Omega_{k}, P_{k, n}\right\}} & \sum_{k=K_{1}+1}^{K} \sum_{n \in \Omega_{k}} r_{k, n} \\
\text { subject to } & \sum_{n \in \Omega_{k}} r_{k, n} \geq R_{k}, k=1, \ldots, K_{1} \\
& \sum_{k=1}^{K} \sum_{n \in \Omega_{k}} P_{k, n}=P_{T} \\
& P_{k, n} \geq 0, \forall k, n \\
& \Omega_{1} \cup \Omega_{2} \cup \ldots \cup \Omega_{K} \subseteq\{1,2, \ldots, N\} \\
& \Omega_{1}, \Omega_{2}, \ldots, \Omega_{K} \text { are disjoint }
\end{aligned}
$$

where $\Omega_{k}$ is the set of subcarriers assigned to user $k$. $\Omega_{k}$ 's must be mutually exclusive since each subcarrier is allowed to be used by one user only. In general, it is necessary to share the same subcarrier among multiple users in order to achieve the multiuser capacity region [13]. This suggests that superposition coding together with high complexity decoding should be used. However, there is only a small range of frequency with overlapping sharing according to [13] when optimal power control is used. We therefore focus on mutually exclusive subcarrier assignment schemes, which can also simplify transmitter and receiver implementation for practical systems.

\section{Time-Sharing Based Optimal Subcarrier And Power Allocation}

Finding the optimization variables $\Omega_{k}$ and $P_{k, n}$ for all $k$ and $n$ in (2) is a mixed integer programming problem. In the system with $K$ users and $N$ subcarriers, there are $K^{N}$ possible subcarrier assignments since each subcarrier can be used by one user only. For each subcarrier assignment, the total power will be allocated to meet the individual rate requirement for each DC user and at the same time to maximize the sum-rate of the NDC users. The subcarrier assignment together with its associated power allocation that results in the largest sum-rate while satisfying all the constraints is the optimal solution.

An approach to make the problem more tractable is to relax the constraint that each subcarrier is used by one user only. We introduce a sharing factor $\rho_{k, n} \in[0,1]$ indicating the portion of time that subcarrier $n$ is assigned to user $k$ during each transmission frame. This time-sharing technique was first proposed in [3] and has been frequently used in the context of subcarrier assignment in multiuser OFDM systems to convert a mixed integer 
programming problem into a convex optimization problem [5], [6], [9], [10]. In addition, we introduce a variable $s_{k, n}$ and define it as $s_{k, n}=\rho_{k, n} P_{k, n}$ for all $k$ and $n$. Clearly, $s_{k, n}$ becomes the actual amount of power allocated to user $k$ on subcarrier $n$, whereas $P_{k, n}$ is the power as if subcarrier $n$ is occupied by user $k$ only. If $\rho_{k, n}=0$, we always have $s_{k, n}=0$ but $P_{k, n}$ is not necessarily equal to zero. For notation brevity, we let $\alpha_{k, n}=\left|h_{k, n}\right|^{2} /\left(\Gamma_{k} N_{0} B / N\right)$ for all $k$ and $n$, which is called the effective channel-to-noise ratio (CNR) of user $k$ on subcarrier $n$. Here, for the purpose of generality, the subindex $k$ is added to the SNR gap $\Gamma$ to include the case when each user has different BER requirements if adaptive modulation and coding is used. With the aid of time-sharing factors $\rho_{k, n}$ we now readily transform the original problem (2) into:

$$
\begin{aligned}
\max _{\left\{\rho_{k, n}, s_{k, n}\right\}} & \sum_{k=K_{1}+1}^{K} \sum_{n=1}^{N} \rho_{k, n} \log _{2}\left(1+\frac{s_{k, n} \alpha_{k, n}}{\rho_{k, n}}\right) \\
\text { subject to } & \sum_{n=1}^{N} \rho_{k, n} \log _{2}\left(1+\frac{s_{k, n} \alpha_{k, n}}{\rho_{k, n}}\right) \geq R_{k}, 1 \leq k \leq K_{1} \\
& \sum_{k=1}^{K} \sum_{n=1}^{N} s_{k, n}=P_{T} \\
& \sum_{k=1}^{K} \rho_{k, n}=1, \forall n \\
& s_{k, n} \geq 0,0 \leq \rho_{k, n} \leq 1, \forall k, n .
\end{aligned}
$$

The objective function (3) is a sum of functions of the form $f\left(\rho_{k, n}, s_{k, n}\right)=\rho_{k, n} \log _{2}(1+$ $\left.C s_{k, n} / \rho_{k, n}\right)$, where $C$ is some positive constant. By evaluating the Hessian matrix of $f\left(\rho_{k, n}, s_{k, n}\right)$ at $\rho_{k, n}$ and $s_{k, n}$, we can prove that $f\left(\rho_{k, n}, s_{k, n}\right)$ is concave [14]. Thus, the objective function is also concave since any positive linear combination of concave functions is concave. Moreover, since the inequality constraint functions in (4) are convex and the constraints in (5)-(7) are all affine, the feasible set of this optimization problem is convex. Therefore, the problem in (3)-(7) is a convex optimization problem and there exists a unique optimal solution, which can be obtained in polynomial time. In the following we derive some desirable properties of the optimal solution. 
The Lagrangian of the above problem is given by

$$
\begin{aligned}
J_{1}\left(\left\{\rho_{k, n}\right\},\left\{s_{k, n}\right\}, \boldsymbol{\beta}, \mu, \boldsymbol{v}\right)= & \sum_{k=K_{1}+1}^{K} \sum_{n=1}^{N} \rho_{k, n} \log _{2}\left(1+\frac{s_{k, n} \alpha_{k, n}}{\rho_{k, n}}\right)+ \\
& \sum_{k=1}^{K_{1}} \beta_{k}\left[\sum_{n=1}^{N} \rho_{k, n} \log _{2}\left(1+\frac{s_{k, n} \alpha_{k, n}}{\rho_{k, n}}\right)-R_{k}\right]+ \\
& \mu\left(P_{T}-\sum_{k=1}^{K} \sum_{n=1}^{N} s_{k, n}\right)+\sum_{n=1}^{N} v_{n}\left(1-\sum_{k=1}^{K} \rho_{k, n}\right),
\end{aligned}
$$

where $\boldsymbol{\beta}=\left(\beta_{1}, \ldots, \beta_{K_{1}}\right) \succeq 0, \mu \geq 0$ and $\boldsymbol{v}=\left(v_{1}, \ldots, v_{N}\right)$ are the Lagrange multipliers for the constraints (4), (5) and (6), respectively. The boundary constraints (7) will be absorbed in the Karush-Kuhn-Tucker (KKT) conditions [14] as shown later. Let $\rho_{k, n}^{*}$ and $s_{k, n}^{*}$ denote the optimal solution, if it exists, for $1 \leq k \leq K, 1 \leq n \leq N$. Applying the KKT conditions, we can obtain the necessary and sufficient conditions for $\rho_{k, n}^{*}$ and $s_{k, n}^{*}$. Specifically, $\rho_{k, n}^{*}$ and $s_{k, n}^{*}$ should satisfy the following equations:

$$
\begin{gathered}
\frac{\partial J_{1}(\ldots)}{\partial s_{k, n}^{*}}\left\{\begin{array}{l}
=0, s_{k, n}^{*}>0 \\
<0, s_{k, n}^{*}=0
\end{array}, \quad \forall k, n\right. \\
\frac{\partial J_{1}(\ldots)}{\partial \rho_{k, n}^{*}}\left\{\begin{array}{l}
<0, \rho_{k, n}^{*}=0 \\
=0,0<\rho_{k, n}^{*}<1, \quad \forall k, n \\
>0, \rho_{k, n}^{*}=1
\end{array}\right. \\
\beta_{k}\left[\sum_{n=1}^{N} \rho_{k, n}^{*} \log _{2}\left(1+\frac{s_{k, n}^{*} \alpha_{k, n}}{\rho_{k, n}^{*}}\right)-R_{k}\right]=0, \quad 1 \leq k \leq K_{1} .
\end{gathered}
$$

\section{A. Optimal Power Allocation for Given Subcarrier Assignment}

In this subsection, we present the optimal power distribution when subcarrier assignment is given.

Let $\left\{\rho_{k, n}\right\}$ be any given subcarrier assignment scheme. Differentiating the Lagrangian in (8) with respect to $s_{k, n}$ and substituting the result into the KKT condition (9), we obtain:

$$
P_{k, n}^{*}=\frac{s_{k, n}^{*}}{\rho_{k, n}}=\left(\frac{\beta_{k}}{\mu \ln 2}-\frac{1}{\alpha_{k, n}}\right)^{+}
$$

for $k=1, \ldots, K_{1}$ and $n=1, \ldots, N$, and

$$
P_{k, n}^{*}=\frac{s_{k, n}^{*}}{\rho_{k, n}}=\left(\frac{1}{\mu \ln 2}-\frac{1}{\alpha_{k, n}}\right)^{+}
$$

for $k=K_{1}+1, \ldots, K$ and $n=1, \ldots, N$. Here, $(x)^{+} \triangleq \max (0, x)$. 
Equations (12) and (13) clearly show that the optimal power allocation follows the standard water-filling approach, except that the allocated power is only on for $\rho_{k, n}$ portion of time. For each user, more power will be allocated to the subcarriers with higher CNRs and vice versa. But the water levels of different users can be different. Specifically, the water level of each DC user is given by $L_{k}=\beta_{k} /(\mu \ln 2)$, for $k=1, \ldots, K_{1}$, and it should ensure the basic rate requirement $R_{k}$ in (4). Substituting (12) into the KKT condition (11) and in view of $\beta_{k} \neq 0$, we obtain the closed-form expression for $L_{k}$ given by:

$$
L_{k}=\left[\frac{2^{R_{k}}}{\prod_{n \in \Omega_{k}}\left(\alpha_{k, n}\right)^{\rho_{k, n}}}\right]^{1 / \sum_{n \in \Omega_{k}} \rho_{k, n}},
$$

where $\Omega_{k}$ is the set of subcarriers that is assigned to user $k$ with $\rho_{k, n}>0$ and satisfies $\alpha_{k, n}>1 / L_{k}$. In the case where the given subcarrier assignment is mutually exclusive, i.e., all the $\rho_{k, n}$ 's only take one or zero, the water levels $L_{k}$ can be re-expressed as

$$
L_{k}=\left(\frac{2^{R_{k}}}{\prod_{n^{\prime}=1}^{g_{k}} \alpha_{k, n^{\prime}}}\right)^{1 / g_{k}},
$$

where $\alpha_{k, 1^{\prime}} \geq \alpha_{k, 2^{\prime}} \geq \ldots, \geq \alpha_{k,\left|\Omega_{k}\right|^{\prime}}$ are the ordered CNRs of the $k$-th DC user on its allocated subcarrier set $\Omega_{k}$, and $g_{k}\left(\leq\left|\Omega_{k}\right|\right)$ is the largest integer satisfying $\alpha_{k, g_{k}^{\prime}}>1 / L_{k}$. The water level of all the NDC users, on the other hand, is observed from (13) to be the same and is given by $L_{0}=1 /(\mu \ln 2)$. Let $P_{\mathrm{DC}, T}=\sum_{k=1}^{K_{1}} \sum_{n=1}^{N} \rho_{k, n} P_{k, n}^{*}$ represent the actual total power consumed by the $K_{1}$ DC users. Then the water level of NDC users, $L_{0}$, can be obtained numerically by using the total power constraint $P_{\mathrm{DC}, T}+\sum_{k=K_{1}+1}^{K} \sum_{n=1}^{N} \rho_{k, n} P_{k, n}^{*}=P_{T}$.

Fig. 2 illustrates the optimal power distribution based on multi-level water-filling in a multiuser OFDM system with $K=4$ users and $N=8$ subcarriers. In the figure, the height of each blank region represents the inverse of the channel-to-noise ratio and the height of shadowed ones is the allocated power. The water levels of the two DC users, $k=1$ and $k=2$, are given by $L_{1}$ and $L_{2}$, respectively, and they are determined explicitly by the basic rate targets. The water level of the two NDC users, $k=3$ and $k=4$, is given by $L_{0}$, and it depends on the total available power after the subtraction of the power consumed by DC users. This interpretation on the determination of water levels implicitly imposes higher priorities on DC users, for which the basic rate targets must be guaranteed all the time.

\section{B. Optimal Subcarrier Assignment}

The subcarriers and power should be allocated jointly to achieve the optimal solution of the problem formulated in (3)-(7). The previous subsection discussed the analytical expressions of 
the optimal power allocation for a given subcarrier assignment. In this subsection, we derive the optimal strategy for subcarrier assignment assuming the power allocation is optimized.

Taking the partial derivative of the Lagrangian in (8) with respect to $\rho_{k, n}$, we have:

$$
\frac{\partial J_{1}(\ldots)}{\partial \rho_{k, n}}=\tilde{\beta}_{k}\left[\log _{2}\left(1+\frac{s_{k, n} \alpha_{k, n}}{\rho_{k, n}}\right)-\frac{s_{k, n} \alpha_{k, n}}{\ln 2\left(\rho_{k, n}+s_{k, n} \alpha_{k, n}\right)}\right]-v_{n},
$$

where $\tilde{\beta}_{k}=\beta_{k}$ for $1 \leq k \leq K_{1}$ and $\tilde{\beta}_{k}=1$ otherwise. Now we substitute the optimal power allocation (12) and (13) into (16) and apply the KKT condition (10), then we get:

$$
\rho_{k, n}^{*}=\left\{\begin{array}{l}
1, v_{n}<H_{k, n}\left(L_{0}, \tilde{L}_{k}\right) \\
0, v_{n}>H_{k, n}\left(L_{0}, \tilde{L}_{k}\right)
\end{array} .\right.
$$

Here, the variable $\tilde{L}_{k}$, for $k=1, \ldots, K$ is defined as $\tilde{L}_{k}=L_{k}$ for $1 \leq k \leq K_{1}$ and $\tilde{L}_{k}=L_{0}$ otherwise, and the function $H_{k, n}\left(L_{0}, \tilde{L}_{k}\right)$ is given by:

$$
H_{k, n}\left(L_{0}, \tilde{L}_{k}\right)=\frac{\tilde{L}_{k}}{L_{0}}\left\{\left[\log _{2}\left(\alpha_{k, n} \tilde{L}_{k}\right)\right]^{+}-\frac{1}{\ln 2}\left(1-\frac{1}{\alpha_{k, n} \tilde{L}_{k}}\right)^{+}\right\} .
$$

Due to the constraint in (6), we conclude that the optimal subcarrier assignment is decomposed into $N$ independent problems. That is, for each subcarrier $n$, if $H_{k, n}\left(L_{0}, \tilde{L}_{k}\right)$ 's, for $k=$ $1, \ldots, K$, are all distinct, then only the user with the largest $H_{k, n}\left(L_{0}, \tilde{L}_{k}\right)$ can use that subcarrier. In other words, we have:

$$
\rho_{k^{\prime}(n), n}^{*}=1, \rho_{k, n}^{*}=0, \quad \forall k \neq k^{\prime}(n)
$$

where $k^{\prime}(n)=\arg \max _{1 \leq k \leq K} H_{k, n}\left(L_{0}, \tilde{L}_{k}\right)$. Hence, it follows that for a given set of water levels $\left\{L_{0}, L_{1}, \ldots, L_{K_{1}}\right\}$, or equivalently, a given set of Lagrange multipliers $\{\boldsymbol{\beta}, \mu\}$, we can determine the optimal subcarrier allocation using (18). However, the optimal solution obtained may not satisfy the individual rate constraint (4) and the total power constraint (5).

Function $H_{k, n}\left(L_{0}, \tilde{L}_{k}\right)$ in (17) plays a key role in finding the optimal solution of subcarrier and power allocation. We now take a closer look at its features. Firstly, by differentiating $H_{k, n}\left(L_{0}, \tilde{L}_{k}\right)$ with respect to $\alpha_{k, n}$ at $\alpha_{k, n}>1 / \tilde{L}_{k}$, it is seen that $H_{k, n}\left(L_{0}, \tilde{L}_{k}\right)$ is monotonically increasing in $\alpha_{k, n}$. As a result, for each subcarrier, the user with a larger CNR is more likely to be assigned this subcarrier. In the extreme case where DC users are absent (i.e. $K_{1}=0$ ), each subcarrier will be assigned to the user with the largest CNR. This agrees with the previous result in [4]. In the general case, the candidate pool for accessing each subcarrier consists of all the $K_{1}$ DC users and the only one NDC user with the largest CNR. This consequently suggests that the exhaustive search for optimal subcarrier assignment in the original problem (2) has a complexity of $\left(K_{1}+1\right)^{N}$ rather than $K^{N}$. Secondly, we also 
observe that $H_{k, n}\left(L_{0}, \tilde{L}_{k}\right)$ is a non-decreasing function of $L_{k}$ and $L_{0}$, respectively, when $1 \leq k \leq K_{1}$ and $K_{1}<k \leq K$. This is obtained by differentiating $H_{k, n}\left(L_{0}, \tilde{L}_{k}\right)$ with respect to $L_{k}$ and $L_{0}$ for $1 \leq k \leq K_{1}$ and $K_{1}<k \leq K$, respectively. Thus, increasing the water level $L_{k}$ of DC user $k$ increases its chance to occupy more subcarriers. Similarly, increasing the water level $L_{0}$ of NDC users allow them to access more subcarriers.

Using the above observations, we propose in the next subsection an iterative algorithm to find the optimal water levels and determine the corresponding subcarrier assignment so that all the rate and power constraints are satisfied.

\section{Iterative Algorithm}

The thrust of the algorithm is to obtain the set of optimal water levels $\left\{L_{0}, L_{1}, \ldots, L_{K_{1}}\right\}$ using two nested loops. The outer loop varies $L_{0}$ to meet the total power constraint. The inner loop searches $\left\{L_{1}, \ldots, L_{K_{1}}\right\}$ and determines the optimal $\rho_{k, n}$ for all $k$ and $n$ at a given value of $L_{0}$ to satisfy the basic rate requirement for every DC user. The algorithm is outlined as follows.

\section{Optimal Subcarrier Assignment Algorithm}

\section{Main function}

a) Set $L_{\mathrm{LB}}=L_{\mathrm{UB}}=\min _{\substack{K_{1}<k \leq K \\ 1 \leq n \leq N}}\left\{1 / \alpha_{k, n}\right\}-\Delta 1$

b) Find the optimal $\left\{L_{k}, \rho_{k, n}\right\}$ at $L_{0}=L_{\mathrm{UB}}$

Compute the actual power consumption $P_{T}^{\prime}$ using (12) and (13)

if $P_{T}^{\prime}<P_{T}$, update $L_{\mathrm{UB}}=2 L_{\mathrm{UB}}$, and repeat Step b)

else, go to Step c)

c) Find the optimal $\left\{L_{k}, \rho_{k, n}\right\}$ at $L_{0}=\left(L_{\mathrm{LB}}+L_{\mathrm{UB}}\right) / 2$

Compute $P_{T}^{\prime}$ using (12) and (13)

if $P_{T}^{\prime}>P_{T}$, let $L_{\mathrm{UB}}=L_{0}$

elseif $P_{T}^{\prime}<P_{T}$, let $L_{\mathrm{LB}}=L_{0}$

Repeat Step c) until $P_{T}^{\prime}=P_{T}$

Function: find the optimal $\left\{L_{k}, \rho_{k, n}\right\}$ at a given $L_{0}$

1) Set $L_{k}$ using (15) with $\Omega_{k}=\{1, \ldots, N\}$, for $k=1, \ldots, K_{1}$

Compute $H_{k, n}$ using (17), $\forall k, n$

Obtain $\rho_{k, n}$ using (18), $\forall k, n$

\footnotetext{
${ }^{1} \Delta$ is a very small number.
} 
2) Compute $R_{k}^{\prime}=\sum_{n=1}^{N} \rho_{k, n}\left[\log _{2}\left(L_{k} \alpha_{k, n}\right)\right]^{+}$for $k=1, \ldots, K_{1}$

3a) Find $k^{*}$ with $R_{k^{*}}^{\prime}<R_{k^{*}}$ and $R_{k^{*}}^{\prime}-R_{k^{*}} \leq R_{k}^{\prime}-R_{k}$ for all $1 \leq k \leq K_{1}$

3b) Find the subcarrier set $\mathcal{A}_{k^{*}}=\left\{n \mid \rho_{k^{*}, n}<1\right\}$ for the found $k^{*}$ for each $n \in \mathcal{A}_{k^{*}}$

Let $k(n)=\operatorname{argmax}_{1 \leq k \leq K} H_{k, n}$ and obtain $L_{k^{*}}(n)$ such that $H_{k^{*}, n}=(1+\Delta) H_{k(n), n}$

3c) while $R_{k^{*}}^{\prime}<R_{k^{*}}$ for the found $k^{*}$

Denote $m=\arg \min _{n \in \mathcal{A}_{k^{*}}} L_{k^{*}}(n)$

Update $L_{k^{*}}=L_{k^{*}}(m)$

Update $\rho_{k^{*}, m}=1, \rho_{k, m}=0$ for $\forall k \neq k^{*}$, and $\mathcal{A}_{k^{*}}=\mathcal{A}_{k^{*}}-\{m\}$

Compute $R_{k^{*}}^{\prime}=\sum_{n=1}^{N} \rho_{k^{*}, n}\left[\log _{2}\left(L_{k^{*}} \alpha_{k^{*}, n}\right)\right]^{+}$

3d) if $R_{k^{*}}^{\prime}>R_{k^{*}}$, let $R_{k^{*}}^{\prime}=R_{k^{*}}^{\prime}-\log _{2}\left(L_{k^{*}} \alpha_{k^{*}, m}\right)$

if $R_{k^{*}}^{\prime}<R_{k^{*}}$

Update $\rho_{k^{*}, m}=\left(R_{k^{*}}-R_{k^{*}}^{\prime}\right) / \log _{2}\left(L_{k^{*}} \alpha_{k^{*}, m}\right)$ and $\rho_{k(m), m}=1-\rho_{k^{*}, m}$

elseif $R_{k^{*}}^{\prime}>R_{k^{*}}$

Update $\rho_{k^{*}, m}=0, \rho_{k(m), m}=1$ and compute $L_{k^{*}}$ using (14)

4) Repeat Steps 2)-3) until $R_{k}^{\prime}=R_{k}$ for all $k=1, \ldots, K_{1}$

In the outer loop (main function), we initialize $L_{0}$ to a value slightly below the minimum reciprocal of the CNRs of all NDC users over all subcarriers so that none of the NDC users is assigned any power resource based on (13). By doing this, the subcarrier and power will be initially allocated to all DC users as if NDC users were absent. We then keep increasing $L_{0}$ until the actual total power consumption $P_{T}^{\prime}$ exceeds the total available power $P_{T}$ and an upper bound of $L_{0}$ is obtained. Note that if the number of subcarriers in a practical system is large enough, we can always find at least one subcarrier fully occupied by one NDC user and, therefore, an analytical upper bound of $L_{0}$ can be derived from (13) as $L_{\mathrm{UB}}=P_{T}+\max _{\substack{K_{1}<k \leq K \\ 1 \leq n \leq N}}\left\{1 / \alpha_{k, n}\right\}$. The algorithm then proceeds to use the bisection method to update $L_{0}$ so that $\bar{P}_{T}^{\prime}$ converges to $P_{T}$. The outer loop converges because the actual total power consumption $P_{T}^{\prime}$ increases monotonically with $L_{0}$ given the target data rates for all DC users are satisfied.

The operation in the inner loop (function: find the optimal $\left\{L_{k}, \rho_{k, n}\right\}$ at a given $L_{0}$ ) is similar to the algorithm introduced in [3]. Each $L_{k}$ is first initialized to the minimum water level needed by DC user $k$ to achieve its target data rate, which happens when all the $N$ subcarriers in the system are assigned to this user. We then gradually increase $L_{k}$ for one of 
the DC users until the target data rate for this user is satisfied. Increasing $L_{k}$ is carried out step by step and the increment at each step is the minimum required value allowing only one more subcarrier to be added to this DC user. During this process, there are chances that the newly added subcarrier is not fully needed and should be time-shared with other users. It is also possible that this subcarrier is not needed at all and it only needs to adjust $L_{k}$ using (14). The algorithm then switches to another DC user and repeats the process until the target data rates for all DC users are satisfied. The inner loop converges because for a given DC user $k$, as $L_{k}$ increases, $H_{k, n}$ for all $n$ increases and more $\rho_{k, n}$ become one. Hence the data rate $R_{k}^{\prime}=\sum_{n=1}^{N} \rho_{k, n}\left[\log _{2}\left(L_{k} \alpha_{k, n}\right)\right]^{+}$increases. On the other hand, the rates of some other DC users may drop due to their $\rho_{k, n}$ changing from one to zero. Nevertheless, as all the $L_{k}$ 's increase, the rate of each DC user increases and hence the optimal $L_{k}$ 's can be approached iteratively.

The complexity of the above algorithm lies in the number of iterations needed to update $L_{0}$ in the outer loop and the number of iterations to update $\left\{L_{k}, \rho_{k, n}\right\}$ for each $L_{0}$ in the inner loop. Since $L_{0}$ is searched by the bisection method, it requires $\log _{2}(1 / \epsilon)$ iterations to converge, where $\epsilon$ is the accuracy. The empirical study in the Appendix shows that the exact number of iterations to find the optimal $\left\{L_{k}, \rho_{k, n}\right\}$ for each $L_{0}$ can vary for different values of $L_{0}$ and different CNR realizations, but the averaged total number of iterations required to update the set of water levels $\left\{L_{0}, L_{1}, \ldots, L_{K_{1}}\right\}$ in the whole algorithm can be well approximated by $\mathcal{O}\left(K_{1}^{2} / \sqrt{N} \log _{2}(1 / \epsilon)\right)$. Since the computational load in each iteration is linear in $K N$, the overall complexity of the proposed algorithm is $\mathcal{O}\left(K_{1}^{2} K N^{1 / 2} \log _{2}(1 / \epsilon)\right)$.

\section{Feasibility and Service Outage}

Similar to the delay-limited capacity problem in information theory [2], the constant-rate transmission of DC users considered in this paper can only be guaranteed in a probabilistic manner since the total transmit power is fixed and finite. The service is said to be in an outage if any of the basic rate requirements cannot be satisfied. Thus, the feasibility of the optimization problem in (3)-(7) is directly related to the condition that $P_{T} \geq P_{\mathrm{DC}, \text { min }}$, where $P_{\mathrm{DC}, \text { min }}$ is the minimum total power needed to support all $R_{k}$ 's in the absence of NDC users. Finding $P_{\mathrm{DC} \text {,min }}$ reduces to the margin adaption problem [3]. The algorithm proposed in the previous subsection is able to detect the service outage and obtain the outage probability numerically in an efficient way. Specifically, if $P_{T}^{\prime}$ computed in Step b) of the main function is greater than $P_{T}$ when $L_{\mathrm{UB}}$ is still given by the initial value set in Step a), the algorithm 
will terminate and declare an outage.

When outage occurs, one may ignore all the $K_{1}$ DC users and allocate all subcarrier and power resources to the $K-K_{1}$ NDC users only. Alternatively, one may ignore one or more DC users from the user list so that at the current channel condition the resources are sufficient to provide the basic rates of the remaining DC users. Those ignored DC users may be re-scheduled for transmission at the next transmission frame by a higher layer scheduler. Further analysis on higher layer scheduling is out of the scope of this work.

\section{Subcarrier And power Allocation using Dual Decomposition}

The convex relaxation technique in Section III permits time sharing of each subcarrier. The system model it employs thus differs from the original OFDMA system where only mutually exclusive subcarrier assignment is allowed. As a result, the solution gives an upper bound on the achievable maximum sum-rate of all NDC users under the individual rate requirement for each DC user and the total transmit power constraint. Recently, it is shown in [15] that the duality gap of non-convex resource optimization problems in multicarrier systems is nearly zero if the number of subcarriers is sufficiently large. Thus, the original problem can be solved in the dual domain using decomposition method. Applying this result, the authors in [16] developed efficient algorithms to solve the weighted sum-rate maximization and weighted sum-power minimization problems in the downlink of multiuser OFDM systems. In this section we shall apply the result from [15] and solve our original problem (2) using the dual decomposition method. Note that the subcarrier and power allocation solution in this section provides a lower bound on the maximum sum-rate of all NDC users when all the target rates for DC users and the total power constraint are satisfied.

Define $\mathcal{D}$ as a set of all non-negative $P_{k, n}$ 's for $1 \leq k \leq K$ and $1 \leq n \leq N$ such that for each $n$ only one $P_{k, n}$ is positive. The Lagrange dual function of the problem (2) is given by:

$$
\begin{aligned}
g(\boldsymbol{\beta}, \mu)= & \max _{\left\{P_{k, n}\right\} \in \mathcal{D}} J_{2}\left(\left\{P_{k, n}\right\}, \boldsymbol{\beta}, \mu\right) \\
= & \max _{\left\{P_{k, n}\right\} \in \mathcal{D}}\left[\sum_{k=K_{1}+1}^{K} \sum_{n=1}^{N} r_{k, n}+\sum_{k=1}^{K_{1}} \beta_{k}\left(\sum_{n=1}^{N} r_{k, n}-R_{k}\right)\right. \\
& \left.+\mu\left(P_{T}-\sum_{k=1}^{K} \sum_{n=1}^{N} P_{k, n}\right)\right],
\end{aligned}
$$

where $J_{2}\left(\left\{P_{k, n}\right\}, \boldsymbol{\beta}, \mu\right)$ is the Lagrangian and the dual variables $\{\boldsymbol{\beta}, \mu\}$ are defined in the 
same way as in (8). The dual optimization problem is then formulated as:

$$
\begin{aligned}
\operatorname{minimize} & g(\boldsymbol{\beta}, \mu) \\
\text { subject to } & \boldsymbol{\beta} \succeq 0, \mu \geq 0 .
\end{aligned}
$$

Note that the Lagrangian $J_{2}(\ldots)$ is linear in $\beta_{k}$ and $\mu$ for fixed $P_{k, n}$, and $g(\boldsymbol{\beta}, \mu)$ is the maximum of these linear functions, so the dual problem (20) is convex.

To solve the dual problem, we first decompose the dual function into $N$ independent optimization problems:

$$
g(\boldsymbol{\beta}, \mu)=\sum_{n=1}^{N} g_{n}(\boldsymbol{\beta}, \mu)-\sum_{k=1}^{K_{1}} \beta_{k} R_{k}+\mu P_{T}
$$

where

$$
g_{n}(\boldsymbol{\beta}, \mu)=\max _{\left\{P_{k, n}\right\} \in \mathcal{D}}\left[\sum_{k=K_{1}+1}^{K} r_{k, n}+\sum_{k=1}^{K_{1}} \beta_{k} r_{k, n}-\mu \sum_{k=1}^{K} P_{k, n}\right] .
$$

Suppose subcarrier $n$ is assigned to user $k$. Using the KKT condition similar to (9), the optimal $P_{k, n}^{*}$ that maximizes the object of the max operation in (22) for fixed $\boldsymbol{\beta}$ and $\mu$ can be readily obtained and is given by (12) when $1 \leq k \leq K_{1}$, or (13) when $K_{1}<k \leq K$. Substituting (12) and (13) into (22) and comparing all the $K$ possible user assignments of this subcarrier, we obtain

$$
g_{n}(\boldsymbol{\beta}, \mu)=\max _{1 \leq k \leq K}\left\{\tilde{\beta}_{k}\left[\log _{2}\left(\frac{\alpha_{k, n} \tilde{\beta}_{k}}{\mu \ln 2}\right)\right]^{+}-\mu\left(\frac{\tilde{\beta}_{k}}{\mu \ln 2}-\frac{1}{\alpha_{k, n}}\right)^{+}\right\},
$$

where $\tilde{\beta}_{k}=\beta_{k}$ for $k=1, \ldots, K_{1}$ and $\tilde{\beta}_{k}=1$ for $k=K_{1}+1, \ldots, K$.

Once (23) is solved for all $n$ 's, the dual function $g(\boldsymbol{\beta}, \mu)$ can be obtained using (21). Since it is convex, a gradient-type algorithm can minimize $g(\boldsymbol{\beta}, \mu)$ by updating $\{\boldsymbol{\beta}, \mu\}$ simultaneously along some appropriate search directions, which is guaranteed to converge to the optimal solution. In general, $g(\boldsymbol{\beta}, \mu)$ is not differentiable, and thus its gradient does not exist. Nevertheless, we can resort to the subgradient derived in the following proposition.

Proposition 1: For the dual problem (20) with primal defined in (2), the following is a subgradient of $g(\boldsymbol{\beta}, \mu)$

$$
\begin{aligned}
\Delta \beta_{k} & =\sum_{n=1}^{N} r_{k, n}^{*}-R_{k}, k=1 \ldots, K_{1}, \\
\Delta \mu & =P_{T}-\sum_{k=1}^{N} \sum_{n=1}^{N} P_{k, n}^{*},
\end{aligned}
$$

where $P_{k, n}^{*}$ maximizes the Lagrangian $J_{2}(\ldots)$ over $\mathcal{D}$ at $\boldsymbol{\beta}$ and $\mu$, and $r_{k, n}^{*}=\log _{2}\left(1+P_{k, n}^{*} \alpha_{k, n}\right)$. 
Proof: By definition of $g(\boldsymbol{\beta}, \mu)$ in (19):

$$
\begin{aligned}
g\left(\boldsymbol{\beta}^{\prime}, \mu^{\prime}\right) & \geq \sum_{k=K_{1}+1}^{K} \sum_{n=1}^{N} r_{k, n}^{*}+\sum_{k=1}^{K_{1}} \beta_{k}^{\prime}\left(\sum_{n=1}^{N} r_{k, n}^{*}-R_{k}\right)+\mu^{\prime}\left(P_{T}-\sum_{k=1}^{K} \sum_{n=1}^{N} P_{k, n}^{*}\right) \\
& =g(\boldsymbol{\beta}, \mu)+\sum_{k=1}^{K_{1}}\left(\beta_{k}^{\prime}-\beta_{k}\right)\left(\sum_{n=1}^{N} r_{k, n}^{*}-R_{k}\right)+\left(\mu^{\prime}-\mu\right)\left(P_{T}-\sum_{k=1}^{K} \sum_{n=1}^{N} P_{k, n}^{*}\right) .
\end{aligned}
$$

Proposition 1 is hence proven by using the definition of subgradient.

With the above subgradient, both the subgradient and ellipsoid methods [17] can be used to update $\{\boldsymbol{\beta}, \mu\}$. Here we choose the ellipsoid method which converges in $\mathcal{O}\left(\left(K_{1}+1\right)^{2}\right)$ iterations. The algorithm details can be found in [17]. The following lemma leads to a suitable choice of the initial ellipsoid.

Lemma 1: The optimal dual variables $\left\{\boldsymbol{\beta}^{*}, \mu^{*}\right\}$ must satisfy

$$
\begin{aligned}
& 0 \leq \mu^{*} \leq \mu^{\max }=\frac{1}{\ln 2} \max _{\substack{K_{1}<k \leq K \\
1 \leq n \leq N}}\left\{\alpha_{k, n}\right\}, \\
& 0 \leq \beta_{k}^{*} \leq \beta_{k}^{\max }=\max _{\substack{K_{1}<k \leq K \\
1 \leq n \leq N}}\left\{\alpha_{k, n}\right\}\left[P_{T}+\frac{1}{\min _{1 \leq n \leq N}\left\{\alpha_{k, n}\right\}}\right] .
\end{aligned}
$$

Proof: The dual variables $\left\{\boldsymbol{\beta}^{*}, \mu^{*}\right\}$ must satisfy the KKT conditions in order to be optimal. Taking the partial derivative of $J_{2}(\ldots)$ in (19) with respect to $P_{k, n}$ results in

$$
\frac{\alpha_{k, n}}{\ln 2\left(1+\alpha_{k, n} P_{k, n}\right)}=\mu
$$

if user $k$, for $K_{1}<k \leq K$, is active in subcarrier $n$, or

$$
\frac{\beta_{k} \alpha_{k, n}}{\ln 2\left(1+\alpha_{k, n} P_{k, n}\right)}=\mu
$$

if user $k$, for $1 \leq k \leq K_{1}$, is active in subcarrier $n$. Since $P_{k, n}$ must always satisfy $0 \leq$ $P_{k, n} \leq P_{T}$ due to the power constraint, we obtain the upper bound $\mu^{\max }$ by letting $P_{k, n}=0$ in (24) and the upper bound $\beta_{k}^{\max }$ by substituting $\mu^{\max }$ into (25) and letting $P_{k, n}=P_{T}$.

Using Lemma 1, one may choose an initial ellipsoid that encloses the hyper-cuboid where $\left\{\boldsymbol{\beta}^{*}, \mu^{*}\right\}$ resides, namely, $E\left(\mathbf{A}_{0}, \mathbf{z}_{0}\right)=\left\{\mathbf{x} \mid\left(\mathbf{x}-\mathbf{z}_{0}\right)^{T} \mathbf{A}_{0}^{-1}\left(\mathbf{x}-\mathbf{z}_{0}\right) \leq 1\right\}$, where

$$
\begin{aligned}
\mathbf{A}_{0} & =\operatorname{diag}\left[\left(1+K_{1}\right)\left(\frac{1}{2} \beta_{1}^{\max }\right)^{2}, \cdots,\left(1+K_{1}\right)\left(\frac{1}{2} \beta_{K_{1}}^{\max }\right)^{2},\left(1+K_{1}\right)\left(\frac{1}{2} \mu^{\max }\right)^{2}\right] \\
\mathbf{z}_{0} & =\left[\frac{1}{2} \beta_{1}^{\max }, \cdots, \frac{1}{2} \beta_{K_{1}}^{\max }, \frac{1}{2} \mu^{\max }\right]^{T} .
\end{aligned}
$$

Due to duality gap, after obtaining the optimal dual variables $\left\{\boldsymbol{\beta}^{*}, \mu^{*}\right\}$ that minimize the dual function, it remains to find the optimal primal solutions $\left\{P_{k, n}^{*}\right\}$ that maximize the Lagrangian $J_{2}(\ldots)$ and satisfy all the rate and power constraints in the original problem (2). 
We can solve this by first identifying the subcarrier assignment $\left\{\Omega_{k}^{*}\right\}$ using $(23)$ with $\left\{\boldsymbol{\beta}^{*}, \mu^{*}\right\}$ substituted and then determining the power allocation $\left\{P_{k, n}^{*}\right\}$ using the results derived in Section III-A.

\section{Suboptimal Subcarrier and Power Allocation}

In this section we present a suboptimal allocation algorithm that has a much lower computational cost compared with both the optimal iterative algorithm in Section III-C and the dual update algorithm in Section IV. The idea is to first obtain the subcarrier assignment for the DC users by assuming that the power is equally distributed over all subcarriers and that all the NDC users are absent. After that, the power distribution for each DC user over its assigned subcarrier set is individually refined. The purpose of the refinement is to minimize the power while maintaining the basic transmission rates. At last, the residual subcarriers and power are distributed among the NDC users using the optimal resource allocation algorithm in [4] to maximize the sum-rate. This algorithm is suboptimal because the subcarrier assignment for DC users in the first step is obtained by assuming equal power allocation. The decoupling of subcarrier assignment and power allocation for DC users carried out in the first two steps, though being suboptimal, can greatly simplify the complexity and is often used for resource allocation in multiuser OFDM systems such as [9], [10], [18]

The outline of the proposed suboptimal subcarrier assignment scheme for DC users is presented below.

\section{Suboptimal Subcarrier Allocation Algorithm for DC users}

1) set $R_{k}^{\prime}=0, \Omega_{k}=\emptyset$ for all $k=1, \ldots, K_{1}$ and $\mathcal{A}=\{1,2, \ldots, N\}$

2) while $\mathcal{A} \neq \emptyset$ and $R_{k}^{\prime}<R_{k}$ for any $1 \leq k \leq K_{1}$

a) find $k^{*}$ with $R_{k^{*}}^{\prime}<R_{k^{*}}$ and $R_{k^{*}}^{\prime}-R_{k^{*}} \leq R_{k}^{\prime}-R_{k}$ for all $1 \leq k \leq K_{1}$

b) for the found $k^{*}$, find $n^{*}$ satisfying $\alpha_{k^{*}, n^{*}} \geq \alpha_{k^{*}, n}$ for $n \in \mathcal{A}$

c) for the found $k^{*}$ and $n^{*}$, update $\Omega_{k^{*}}=\Omega_{k^{*}} \cup\left\{n^{*}\right\}, \mathcal{A}=\mathcal{A}-\left\{n^{*}\right\}$ and $R_{k^{*}}^{\prime}=$ $R_{k^{*}}^{\prime}+\log _{2}\left(1+\frac{\alpha_{k^{*}, n^{*}} P_{T}}{N}\right)$

At each iteration of Step 2) in the above algorithm, the DC user whose current data rate $R_{k}^{\prime}$ is the farthest away from its target rate $R_{k}$ will be allowed to pick a new subcarrier from the available subcarrier set. Preferably, the subcarrier with the highest CNR will be chosen.

Upon acquiring $\Omega_{k}$ for $1 \leq k \leq K_{1}$, the power distribution for each DC user is then adjusted using the analytical solution (12) and (15). In the case where $g_{k}<\left|\Omega_{k}\right|$ for some $k$, 
the above suboptimal subcarrier assignment algorithm over-allocates subcarriers to DC user $k$. To efficiently utilize all the wireless resources, the remaining $\left|\Omega_{k}\right|-g_{k}$ subcarriers should be returned to the residual subcarrier set $\mathcal{A}$, which will be distributed among the $K-K_{1}$ NDC users. Let $P_{\mathrm{DC}, T}$ denote the actual power consumption of all the $K_{1} \mathrm{DC}$ users. If $P_{\mathrm{DC}, T}$ is larger than the total power limit $P_{T}$, a service outage occurs. Otherwise, the residual transmit power $P_{T}-P_{\mathrm{DC}, T}$ together with the residual subcarrier set $\mathcal{A}$ are allocated over the $K-K_{1}$ NDC users. Specifically, each subcarrier in $\mathcal{A}$ is assigned to the NDC user with the highest CNR, and the power is distributed over these subcarriers in the form of water-filling (13), where the water level can be determined by $P_{T}-P_{\mathrm{DC}, T}$.

The number of iterations involved in finding the suboptimal $\Omega_{k}$ 's for $k=1, \ldots, K$ is limited by $N$ since $N$ is the total number of subcarriers available. That is, the proposed suboptimal algorithm only performs a fixed number of iterations rather than iterating till convergence. The power allocation for given $\left\{\Omega_{k}\right\}$ has explicit analytical solution as shown in Section III-A and its complexity is linear in $K N$. Therefore, the overall complexity of this suboptimal algorithm is only linear in $K$ and $N$.

\section{NUMERicAl RESULTS}

In this section we present numerical performance results of the proposed optimal and suboptimal resource allocation algorithms. We consider a multiuser OFDM system with $N=$ 64 subcarriers and $K=8$ users. Therein, $K_{1}=4$ users have DC traffic and the others have NDC traffic. For simplicity, we let the rate requirements of all DC users be identical and equal to $R_{\mathrm{DC}} / K_{1}$ bits/OFDM symbol, where $R_{\mathrm{DC}}$ denotes the sum of the basic rates. In all simulations, the channel from the base station to each user terminal is modeled by the HiperLan/2 channel model A [19], which is an 8-tap channel with exponential power delay profile, $20 \mathrm{MHz}$ sampling frequency and $50 \mathrm{~ns}$ rms (root-mean-square) delay spread. The channels for different users are assumed to be independent. We also assume that the path losses from the base station to all user terminals are the same. The average channel gain on each subcarrier is normalized. The system total transmit SNR is defined as $P_{T} /\left(N_{0} B\right)$. The SNR gap in the rate function (11) is set to $6.6(8.2 \mathrm{~dB})$ for both DC users and NDC users. In practice, when uncoded QAM constellation is used the SNR gap of $8.2 \mathrm{~dB}$ corresponds to a BER requirement of $10^{-5}$.

To evaluate the performance of the three proposed adaptive resource allocation algorithms, we also present the results for two non-adaptive schemes in comparison. In both schemes, 
the subcarrier assignment is predetermined but the power allocation for each user over its predetermined set of subcarriers follows the optimal approach derived in Section III-A. In the first scheme, all the 8 users are treated equally and each is assigned 8 subcarriers. We refer to this scheme as Fixed Subcarrier Assignment with Optimal Power Allocation (FSA-OPA). In the other scheme, DC users are given higher priority than NDC users and each is assigned 12 subcarriers, whereas each NDC user is allocated 4 subcarriers only. This scheme is called Fixed Subcarrier Assignment with Priority and with Optimal Power Allocation (FSAP-OPA). In addition, for both schemes, we let the predetermined subcarriers for each user spread over the entire bandwidth in a comb pattern [20]. This prevents the situation where all subcarriers of a user are in deep fade.

We first compare the performance in terms of service outage behavior. Fig. 3 illustrates the service outage probability versus total transmit SNR when the total target transmission rate of DC users is $R_{\mathrm{DC}}=80$ bits/OFDM symbol. It is first observed that the time-sharing based optimal algorithm and the dual method perform almost identically. This suggests that two algorithms result in almost the same subcarrier assignment solution for DC users. This observation is expected because the duality gap vanishes when $N$ is sufficiently large and, as a result, both the upper bound given by the optimal algorithm with time sharing and the lower bound given by the dual method approach the truly optimal solution. One can also see that the performance loss due to the suboptimal subcarrier assignment in the suboptimal algorithm is marginal. In particular, at a service outage probability of $1 \%$, the SNR loss is within $0.5 \mathrm{~dB}$. In addition, it is seen from Fig. 3 that the proposed adaptive algorithms significantly outperform the two fixed subcarrier assignment (FSA) schemes. At moderate and high SNR regions, the service outage probability is more than an order of magnitude lower. Besides, the FSA scheme with priority outperforms the one without priority as more subcarriers are assigned to DC users in the former.

In Fig. 4, we plot the minimum required total transmit SNR for different $R_{\mathrm{DC}}$ at a given service outage probability of $1 \%$. It is again observed that the optimal algorithm with time sharing and the dual method have almost identical performance. Therefore, only the results of the former will be illustrated hereafter. From Fig. 4 we observe that, for a wide range of $R_{\mathrm{DC}}$ that the multiuser OFDM system can support with $1 \%$ outage probability, the difference on the minimum required SNR between the optimal and suboptimal algorithms is consistently less than $0.5 \mathrm{~dB}$. In particular, as $R_{\mathrm{DC}}$ decreases, the performance of the suboptimal algorithm becomes closer to that of the optimal algorithm. This is expected as the suboptimality of the 
proposed suboptimal algorithm lies only in the subcarrier allocation for DC users. If the rate requirement for DC users is small, the suboptimal algorithm will become nearly optimal. Fig. 4 also shows that, as $R_{\mathrm{DC}}$ increases, the minimum required total SNR of the proposed adaptive algorithms increases at a much lower speed than that of the two FSA schemes.

We next study the achievable transmission rates of the heterogenous multiuser OFDM system with the proposed adaptive subcarrier and power allocation algorithms. Fig. 5 shows the achievable pairs of the basic sum-rate for DC traffic $R_{\mathrm{DC}}$ and the average sum-rate for NDC traffic $\bar{R}_{\mathrm{NDC}}$ at a total transmit SNR of $20 \mathrm{~dB}$. The average sum-rate for NDC traffic $\bar{R}_{\mathrm{NDC}}$ is obtained by averaging the instantaneous sum-rates of NDC users over 500 independent channel realizations. To ensure a service outage probability of $1 \%$ or below, the maximum value of $R_{\mathrm{DC}}$ in our simulation is set to 176 bits/OFDM symbol for the proposed algorithms, and to 112 and 80 bits/OFDM symbols, respectively, for FSAP-OPA and FSA-OPA. The maximum achievable $R_{\mathrm{DC}}$ with an acceptable service outage probability, for example 1\%, at a given total SNR can be obtained from Fig. 4. From Fig. 5 one can observe that, compared with the optimal subcarrier and power allocation algorithm, the loss of the average achievable NDC traffic rate at a given $R_{\mathrm{DC}}$ by using the suboptimal algorithm is within $2 \% \sim 9 \%$. On the other hand, compared with the two FSA schemes, both the proposed optimal and suboptimal adaptive algorithms demonstrate substantially larger achievable rate regions. We also observe that, at the same $R_{\mathrm{DC}}$, the $\bar{R}_{\mathrm{NDC}}$ of FSA-OPA is larger than that of FSAP-OPA. This is because NDC users have fewer subcarriers in FSAP-OPA. However, the maximum $R_{\mathrm{DC}}$ FSAP-OPA can support is larger than that of FSA-OPA.

Finally, we demonstrate the multiuser diversity exploited by our algorithms. We let the number of DC users in the system be fixed at $K_{1}=4$ and vary the number of NDC users between 4 and 16. Fig. 6 presents the average sum-rate $\bar{R}_{\mathrm{NDC}}$ as a function of the number of NDC users at $R_{\mathrm{DC}}=32$ bits/OFDM symbols. Same to Fig. 5, the total transmit SNR is $20 \mathrm{~dB}$ and $\bar{R}_{\mathrm{NDC}}$ is obtained by averaging 500 independent channel realizations. In the two FSA schemes, the subcarrier allocation for DC users is the same as before, but the rest of the subcarriers are all allocated to one NDC user, which is selected in a round-robin fashion at each transmission frame. The values of $\bar{R}_{\mathrm{NDC}}$ for the two FSA schemes remain constant since no multiuser diversity is achieved. On the contrary, $\bar{R}_{\mathrm{NDC}}$ obtained by the proposed adaptive algorithms increases as the number of NDC users increases, which clearly shows the multiuser diversity. In particular, the achievable $\bar{R}_{\mathrm{NDC}}$ of the optimal algorithm is about $110 \%$ and $140 \%$ higher than that of the FSAP-OPA scheme when the system has 4 and 16 
NDC users, respectively.

\section{CONCLUSION AND DiscUSSIONS}

Supporting communication services with diverse QoS requirements in future broadband wireless networks is crucial. This paper considered the resource allocation problem in an OFDM based downlink system that supports simultaneous transmission of users with DC traffic at constant rates and users with NDC traffic at variable rates. We investigated this problem from the physical layer perspective and aimed to maximize the sum-rate of NDC traffic while maintaining individual basic rates of DC traffic for each channel realization under a total power constraint. It was shown that the optimal power allocation over the subcarriers in such a heterogeneous system has the form of multi-level water-filling; moreover, the set of valid user candidates competing for each subcarrier consists of only one NDC user but all DC users. We converted this combinatorial problem with exponential complexity into a convex problem using the time-sharing technique and developed an efficient iterative algorithm with polynomial complexity. We also solved the original problem using dual decomposition method which leads to polynomial complexity as well. To further speed up the resource allocation and make it more suitable for practical systems, we then proposed a suboptimal algorithm whose computation load is only linear in the number of users and subcarriers in the system.

The performance of our algorithms was evaluated in terms of service outage probability, achievable DC and NDC traffic rate pairs, and multiuser diversity. The numerical results showed that the convex relaxation technique with time sharing and the dual decomposition approach obtained almost the same solution and that the suboptimal algorithm has the near optimal performance. Results also demonstrated that the proposed adaptive subcarrier and power allocation algorithms significantly outperform the schemes with adaptive power allocation but fixed subcarrier assignment.

This paper adopted the continuous rate function (1), which greatly helped to derive the insights of optimal resource allocation. If discrete rates are used in practical systems, our algorithms can be modified accordingly. In particular, since the proposed suboptimal algorithm has near-optimal performance at significantly lower complexity, it is more desirable to modify the suboptimal one. For instance, one can obtain the subcarrier assignment using the proposed suboptimal algorithm and then apply the greedy bit loading algorithm for each single user as in [3]. Nevertheless, our continuous rate formulation provides the performance upper bound for systems with discrete rates. 
We have also assumed that the channels from the base station to all the NDC users have the same path loss. By symmetry, our formulation also equalizes the long-term average throughput among all the NDC user. To achieve fairness when their channel path losses are different, we can simply modify our cost function (2) by dividing the channel-to-noise ratio with the path loss. By doing so, the effective channel gains for all NDC users are normalized. Therefore, only the user whose current channel condition is at its peak level will be selected to compete with DC users for each subcarrier. This is similar to the concept of "riding on the channel peak" in opportunistic scheduling.

\section{APPENDIX}

\section{EMPIRICAL STUDY ON THE CONVERGENCE SPEED OF THE ITERATIVE ALGORITHM IN}

\section{SECTION III-C}

Simulation settings: $P_{T}=100,2$ NDC users, $R_{k}=16$ bits/ OFDM symbol for $1 \leq k \leq$ $K_{1}, K_{1} \in\{1, \ldots, 12\}, N \in\{16,32,64,128\} . \alpha_{k, n}=N\left|h_{k, n}\right|^{2}$ are randomly generated with $h_{k, n}$ modeled as complex Gaussian variables of zero mean and unit variance and independent for all $k$ and $n$. The accuracy of bisection-searching $L_{0}$ is set to $\epsilon=10^{-7}$, and it leads to 26 iterations in the main function throughout this simulation study. The parameter $\Delta$ in Step $3 b$ ) of the inner function is set to 0.005 . Note that it typically takes very few iterations in the while loop of Step 3c) in the inner function to find the $L_{k^{*}}$ for DC user $k^{*}$ that meets its rate requirement. Thus we choose to count the number of times it repeats for Steps 2)-3) as the number of iterations to update $\left\{L_{k}, \rho_{k, n}\right\}$ at a given $L_{0}$. Fig. 7 shows the snapshot of iterations to update $\left\{L_{k}, \rho_{k, n}\right\}$ at each updating step of $L_{0}$ for three random channel realizations. The number of iterations varies for different $L_{0}$ and different channel realizations and, in general, more iterations are needed when $K_{1}$ increases. To extract the rules on how the number of iterations change with $N$ and $K_{1}$, we plot in Fig. 8 the averaged total iterations needed to find the set of optimal solutions $\left\{L_{0}, L_{k}, \rho_{k, n}\right\}$, where each value is obtained by averaging over 20 independent channel realizations. For comparison we also plot the curves generated using the analytical expression $c K_{1}^{2} / \sqrt{N}$ with the constant $c$ being $c=26 \times 5.1=132.6$. It is observed that the analytical expression provides a very good approximation on the shape of the simulated curves. Therefore, we conclude that the proposed time-sharing based optimal subcarrier assignment algorithm converges in $\mathcal{O}\left(K_{1}^{2} / \sqrt{N} \log _{2}(1 / \epsilon)\right)$ iterations. 


\section{REFERENCES}

[1] A. J. Goldsmith and P. Varaiya, "Capacity of fading channels with channel side information," IEEE Trans. on Infor. Theory, vol. 43, no. 6, pp. 1986-1992, Nov. 1997.

[2] G. Caire, G. Taricco, and E. Biglieri, "Optimal power control over fading channels," IEEE Trans. on Infor. Theory, vol. 45, no. 5, pp. 1468-1489, July 1999.

[3] C. Y. Wong, R. S. Cheng, K. B. Letaief, and R. D. Murch, "Multiuser OFDM with adaptive subcarrier, bit and power allocation," IEEE Journal on Selected Areas in Comm., vol. 17, no. 10, pp. 1747-1758, Oct. 1999.

[4] J. Jang and K. B. Lee, "Transmit power adaptation for multiuser OFDM systems," IEEE Journal on Selected Areas in Comm., vol. 21, no. 2, pp. 171-178, Feb. 2003.

[5] P. W. C. Chan and R. S. Cheng, "Optimal power allocation in zero-forcing MIMO-OFDM downlink with multiuser diversity," in Proc. of IST Mobile \& Wireless Communications Summit, Dresden, June 2005.

[6] Y. M. Tsang and R. S. Cheng, "Optimal resouce allocation in SDMA/MIMO/OFDM systems under QoS and power constraints," in Proc. of IEEE WCNC, 2004.

[7] J. M. Cioffi, "Digital communications," EE379 Course Reader, Stanford University, 2003.

[8] Y. J. Zhang and K. B. Letaief, "An efficient resource allocation scheme for spatial multiuser access in MIMO/OFDM systems," IEEE Trans. Comm., vol. 53, no. 1, pp. 107-116, Jan. 2005.

[9] W. Rhee and J. M. Cioffi, "Increase in capacity of multiuser OFDM system using dynamic subchannel allocation," in Proc. of IEEE VTC, 2000.

[10] Z. Shen, J. G. Andrews, and B. L. Evans, "Adaptive resource allocation in multiuser OFDM systems with proportional fairness," IEEE Trans. on Wireless Comm., vol. 4, no. 6, pp. 2726-2737, Nov. 2005.

[11] G. Song and Y. Li, "Cross-layer optimization for OFDM wireless networks: Part II: Algorithm development," IEEE Trans. on Wireless Comm., vol. 4, no. 2, pp. 625-634, March 2005.

[12] M. Anas, K. Kim, S. Shin, and K. Kim, "QoS aware power allocation for combined guaranteed performance and best effort users in OFDMA systems," in Proc. International Symposium on Intelligent Signal Processing and Comm. Systems, Nov. 2004.

[13] A. J. Goldsmith and M. Effros, "The capacity region of broadcast channels with intersymbol interference and colored Gaussian noise," IEEE Trans. on Infor. Theory, vol. 47, pp. 219-240, 2001.

[14] S. Boyd and L. Vandenberghe, Convex Optimization. Cambridge University Press, 2004.

[15] W. Yu and R. Lui, "Dual methods for nonconvex spectrum optimization of multicarrier systems," IEEE Trans. on Comm., vol. 54, no. 7, pp. 1310-1322, July 2006.

[16] K. Seong, M. Mhoseni, and J. M. Cioffi, "Optimal resource allocation for OFDMA downlink systems," in Proc. of ISIT'06, Seattle, USA, July 2006.

[17] S. Boyd, “Convex optimization ii," EE364B Course Note, Stanford University. Available at http://www.stanford.edu/class/ee364b/, 2006.

[18] K. Kim, H. Kang, and K. Kim, "Providing quality of service in adaptive resource allocation for OFDMA systems," in Proc. of IEEE VTC'04, vol. 3, May 2004, pp. 1612-1615.

[19] E. N. Committee, "Channel models for HIPERLAN/2 in different indoor scenarios," European Telecom. Standards Institute, Sophia-Antipolis, Valbonne, France, Norme ETSI, doc 3ERIO85B, 1998.

[20] E. Lawrey, "Multiuser OFDM," in Proc. of International Symposium on Signal Processing and its Applications (ISSPA'99), Brisbane, Australia, Aug. 1999. 
Base station transmitter

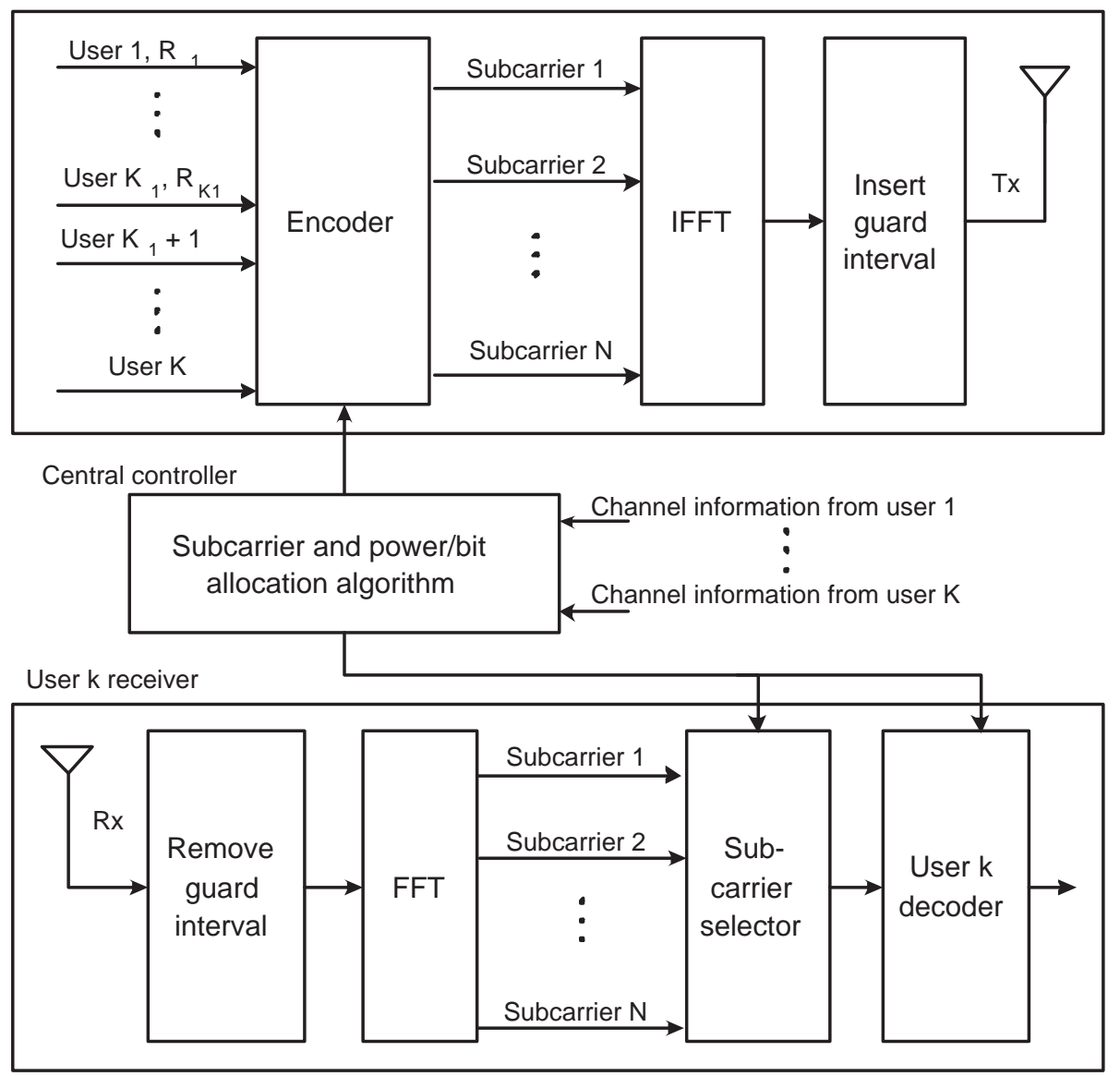

Fig. 1. Block diagram of a downlink multiuser OFDM system 


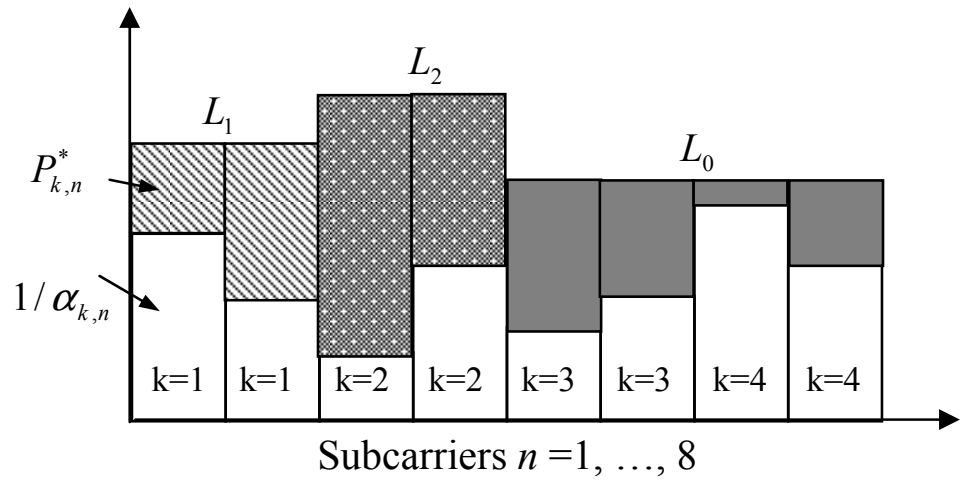

Fig. 2. Illustration of multi-level water-filling for given subcarrier assignment in a multiuser OFDM system with 2 DC users, 2 NDC users and 8 subcarriers.

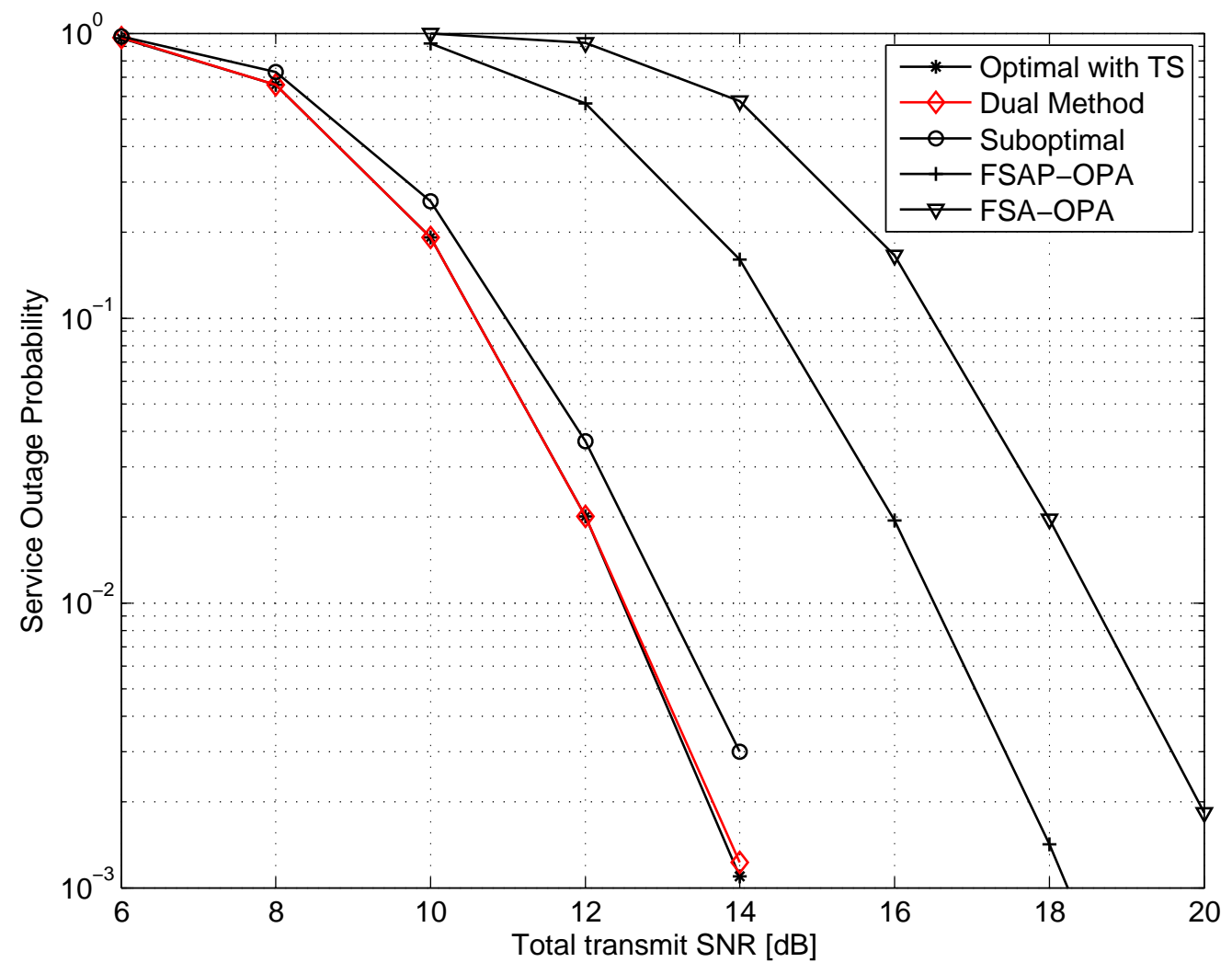

Fig. 3. Service outage probability versus total transmit SNR at $R_{\mathrm{DC}}=80$ bits/OFDM symbol. 


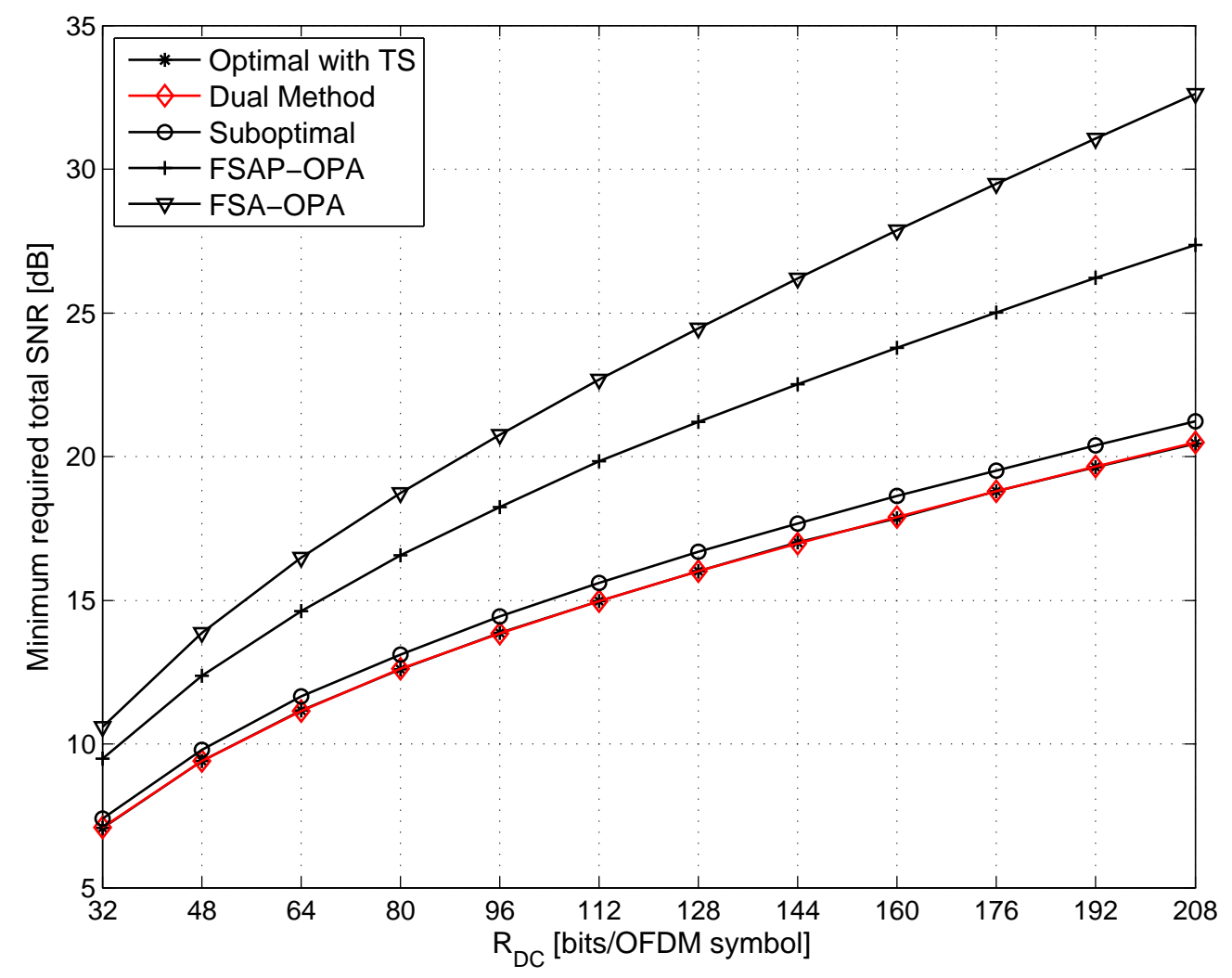

Fig. 4. Minimum required total transmit SNR versus $R_{\mathrm{DC}}$ at a service outage probability of $1 \%$. 


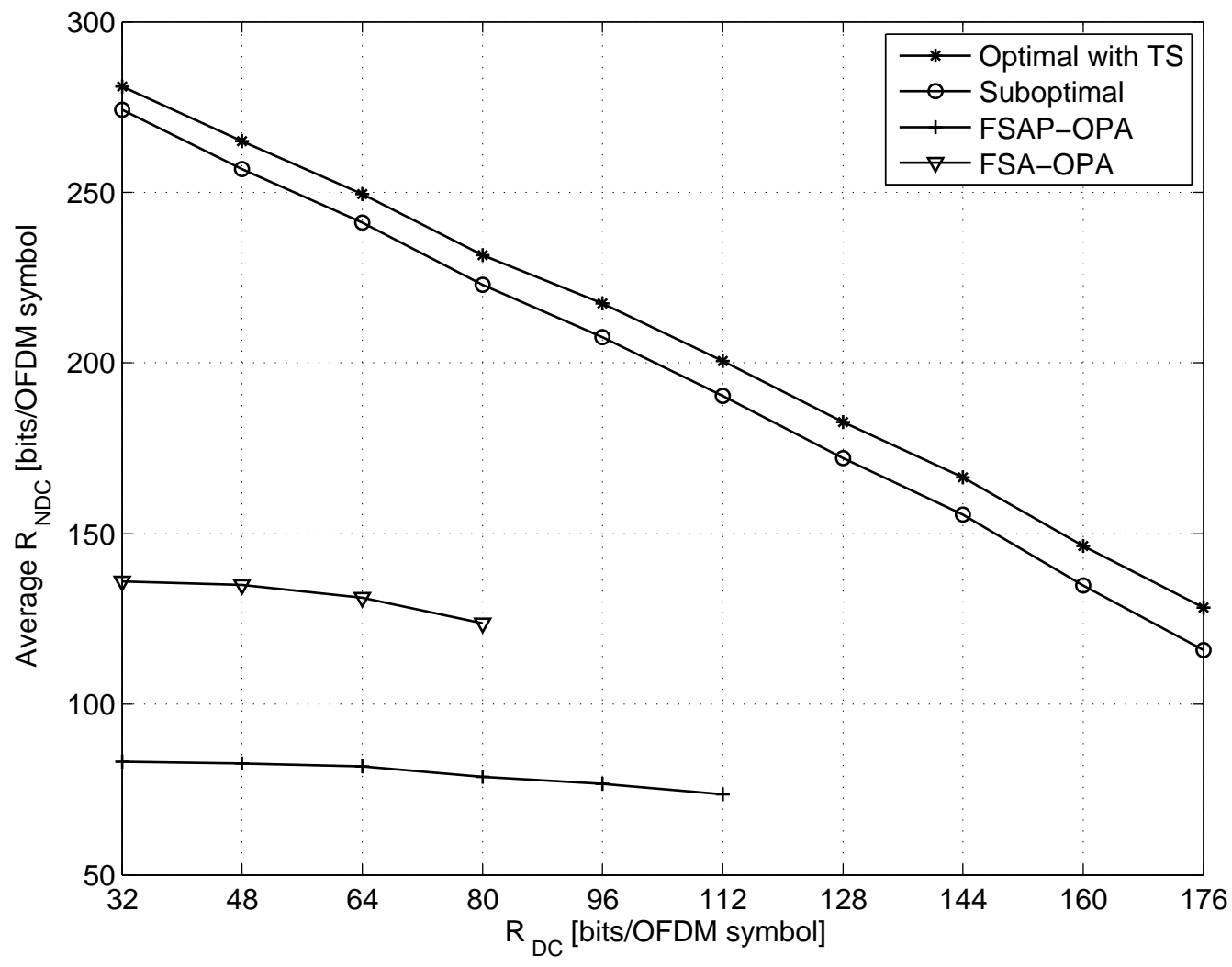

Fig. 5. Achievable $\left(R_{\mathrm{DC}}, \bar{R}_{\mathrm{NDC}}\right)$ rate pairs at a total transmit SNR of $20 \mathrm{~dB}$. 


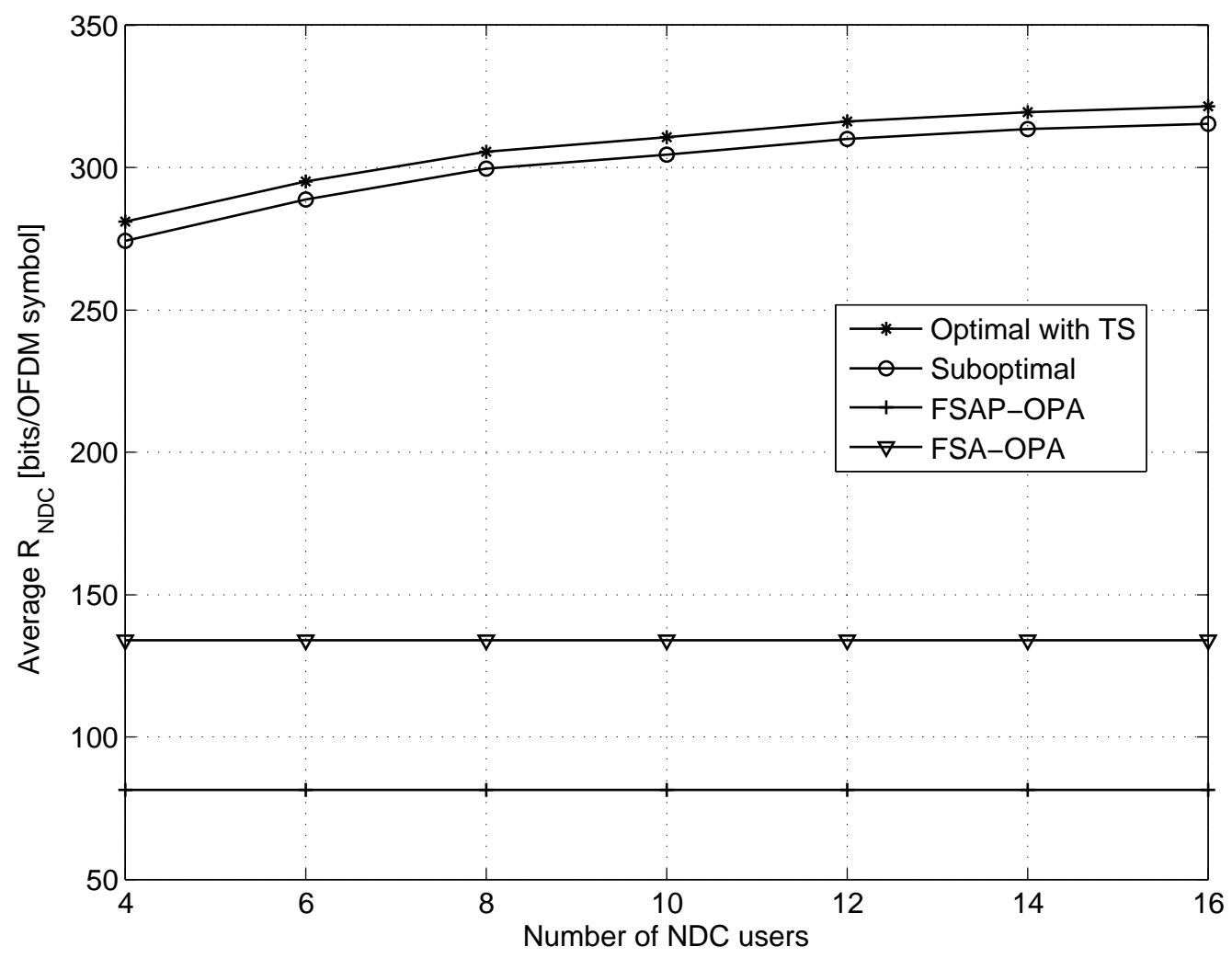

Fig. 6. Achievable $\bar{R}_{\mathrm{NDC}}$ versus the number of NDC users at a total transmit SNR of $20 \mathrm{~dB}$ with 4 DC users.
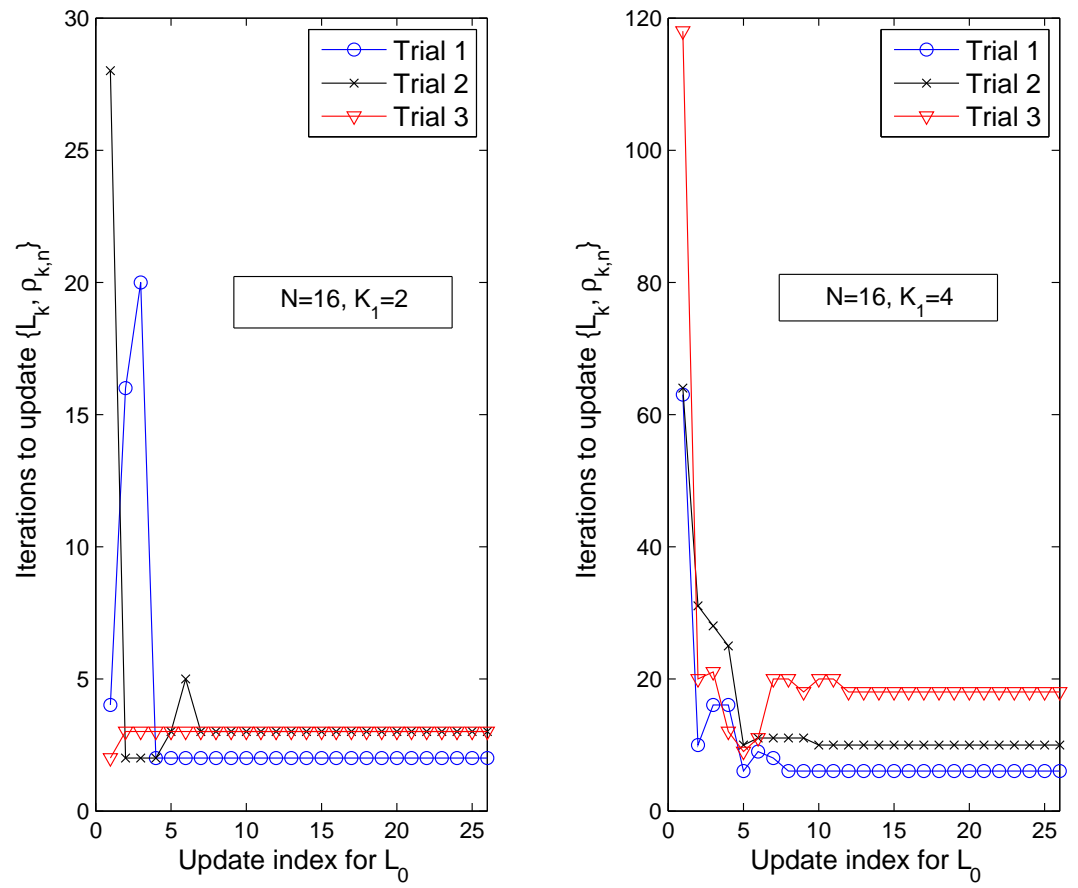

Fig. 7. Iterations required to update $\left\{L_{k}, \rho_{k, n}\right\}$ at each $L_{0}$ update. 


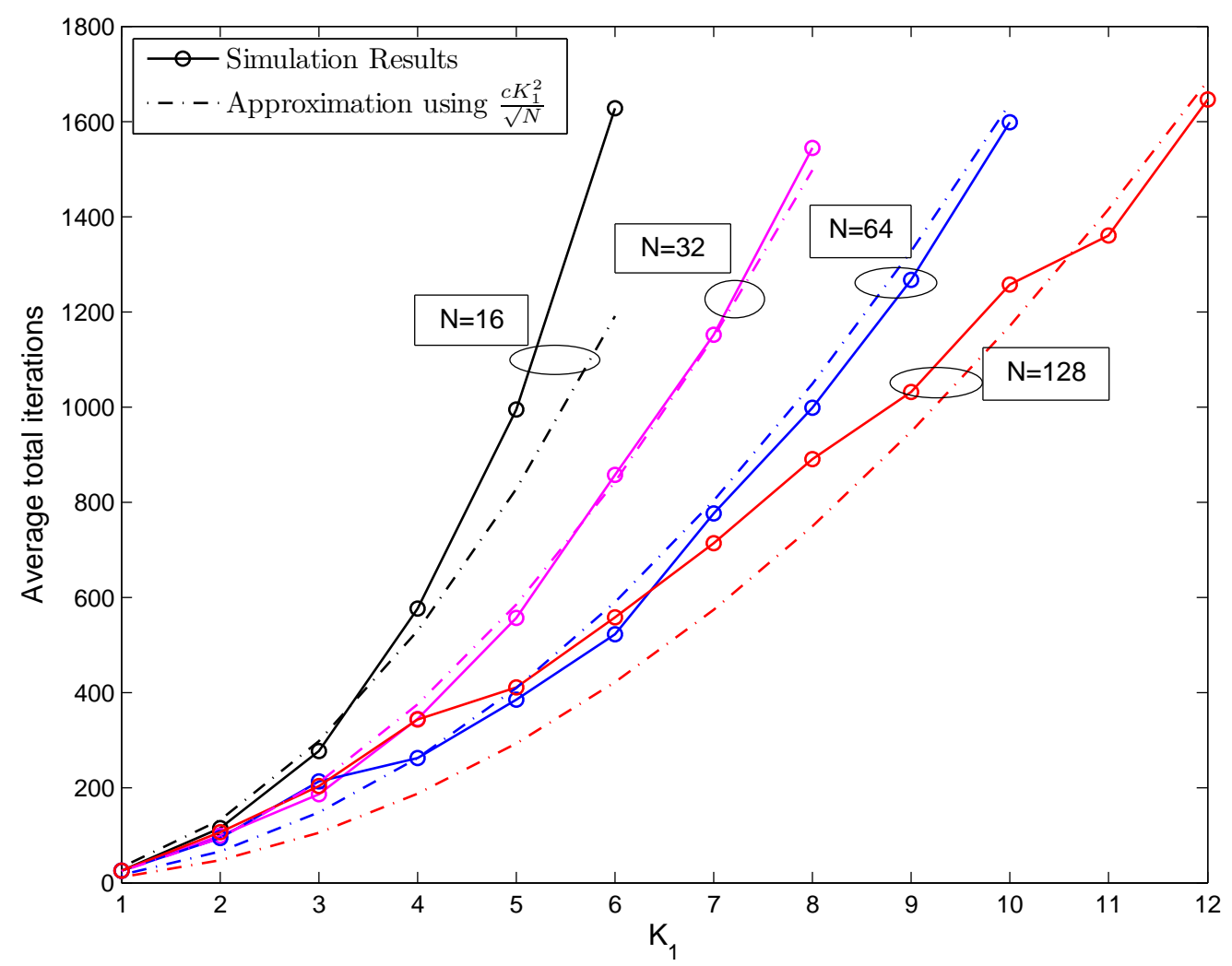

Fig. 8. Total iterations required to update $\left\{L_{0}, L_{k}, \rho_{k, n}\right\}$. 